\title{
Antimuscarinic actions of antihistamines on the heart
}

\author{
Huiling Liu, Qi Zheng \& Jerry M. Farley* \\ Department of Pharmacology and Toxicology, University of Mississippi Medical Center, 2500 N. State St., \\ Jackson, MS, 39216-4505, USA \\ (C) 2006 National Science Council, Taipei
}

Key words: antihistamine, heart, Langendorff, muscarinic receptor, rat

\begin{abstract}
Summary
Antimuscarinic side-effects, which include dry mouth, tachycardia, thickening of mucus possibly sedation, of the antihistamines limited the usefulness of these drugs. The advent of newer agents has reduced the sedative effect of the antihistamine. The data presented here show that one of the newest antihistamines, desloratadine, and a first generation drug, diphenhydramine, are both competitive inhibitors of muscarinic receptor mediated slowing of the heart as measured using a Langendorff preparation. Both agents have apparent sub-micromolar affinities for the muscarinic receptor. Two other agents, cetirizine and fexofenadine, do not interact with muscarinic receptors in the heart at the concentrations used in this study. Structural similarities of the drugs suggest that substitution of a group with a high dipole moment or charge on the side chain nitrogen decreases the binding with muscarinic receptors. We conclude that of the compounds tested fexofenadine and cetirizine have little or no interaction with muscarinic receptors.
\end{abstract}

The newest antihistamines, desloratadine, fexofenadine and cetirizine, are metabolites of the older antihistamines, loratadine, terfenadine and hydroxyzine [1]. All these compounds are selective H1-histamine receptor antagonists. The newer agents also cause less drowsiness than first generation antihistamines (e.g., diphenhydramine) [2]. In addition, the newest compounds do not interact with HERG channels in the heart to cause a prolonged QT syndrome as did terfenadine, the parent compound of fexofenadine [3, 4].

Another potential interaction of antihistamines with cardiac function is at receptors involved in modifying heart rate and contraction. The first generation compounds were known to be competitive inhibitors of muscarinic receptors [5] and caused tachycardia by impairing vagal tone on the heart and xerostomia by inhibiting muscarinic stimulation of salivary function. Desloratadine is reported to inhibit muscarinic receptors at sub-

*To whom correspondence should be addressed. E-mail: jfarley@pharmacology.umsmed.edu micromolar concentrations [6] and therefore could potentially interact on the heart through inhibition of M2-muscarinic receptors. In this report we examine the interaction of antihistamines with muscarinic receptor-induced reduction of cardiac function in a working rat heart model using a Langendorff apparatus.

\section{Methods}

Langendorff

Female Sprague-Dawley rats weighing 300-350 g were used in these experiments. They were housed within the animal facility of University of Mississippi Medical center, and supplied with standard chow and water ad libitum. All experimental procedures were approved by the institutional animal care and use committee.

The basic procedures are similar to those used by Vergely et al. [7]. Rats were anesthetized with phenobarbital sodium (65 $\mathrm{mg} / \mathrm{kg}$ body wt., i.p.), 
and heparinized (500 IU/kg i.p.) to prevent blood coagulation. This protocol was approved by the Institutional Animal Care and Use Committee (IACUC). After opening the chest, the aorta was quickly exposed. The pulmonary artery was then cut and the aorta was immediately cannulated with a perfusion tube (made from a 16 gauge stainless needle) connected to the perfusion system. Retrograde perfusion of heart with Krebs-Henseleit solution $\left(37^{\circ} \mathrm{C}\right)$ was started immediately at a constant flow of $15 \mathrm{ml} / \mathrm{min}$. During the perfusion, the heart was excised midsternally and transferred into a water-jacketed chamber (Radnoti). The heart was allowed to stabilize for $5 \sim 10 \mathrm{~min}$, then a latex balloon $(3 \times 7 \mathrm{~mm}, 0.03 \mathrm{ml}$, Radnoti) filled with physiological saline was inserted into the left atria through the opening in the pulmonary vein into the left ventricle through the mitral valves. The balloon was attached to a PE-90 polyethylene tubing and connected to a pressure transducer. The changes in left ventricular pressure (LVP) and its first derivative $(\mathrm{dP} / \mathrm{dt})$ were recorded through a data-acquisition card (Computer Board PCI-DAS $1602 / 16)$ by a PC running Dasylab 6.0 software (Dasytec). The volume of the balloon in the left ventricle was increased by injection of saline from a syringe connected to the PE90 tubing to set resting wall tension until LVP measurements were maximum and stable. The heart was then perfused for 30-60 min prior to perfusion with any test agents.

The Krebs-Henseleit buffer, consisted of (in mM) $118.5 \mathrm{NaCl}, 4.69 \mathrm{KCl}, 1.18 \mathrm{KH}_{2} \mathrm{PO}_{4}, 1.16$ $\mathrm{MgSO}_{4}, 2.52 \mathrm{CaCl}_{2}, 25.88 \mathrm{NaHCO}_{3}$, and 5.5 glucose, and was freshly prepared and filtered using a $4.5-\mu \mathrm{m}$ pore membrane filter (Millipore; Bedford, MA) before use. The Krebs-Henseleit buffer was bubbled with $95 \% \mathrm{O}_{2}-5 \% \mathrm{CO}_{2}$ to keep a constant pH 7.4 in the perfusion system, and was pre-warmed to $37^{\circ} \mathrm{C}$ in a water-jacketed glass bottle and heating coils (Radnoti). The KrebsHenseleit buffer solution flowed through a waterjacketed bubble trap ( $2 \mathrm{ml} \mathrm{vol})$ immediately before it reached the heart. An outlet tubing with adjustable height was also connected to the bubble trap to maintain the coronary artery perfusion pressure constant at $80 \mathrm{~cm} \mathrm{H}_{2} \mathrm{O}$.

\section{Design}

Acetylcholine and antihistamine containing solutions were perfused into the coronary vessels by switching inflow between tubes of pre-warmed and oxygenated solutions of the desired composition. The basic protocol as shown in Figure 1a was to perfuse the heart with a single concentration of acetylcholine for 3-5 min until a stable change in contraction had occurred and then wash with control solution. When the effect of an antihistamine was tested it was perfused into the heart $5 \mathrm{~min}$ prior to acetylcholine and also during the exposure to acetylcholine. The maximal decrease in contraction induced by acetylcholine was measured and the fractional inhibition of contraction was calculated. Recovery of contraction from the inhibitory effects of acetylcholine upon washout of acetylcholine was required for the data to be accepted as valid. Concentration-response relationships were constructed from the data. The concentrations of acetylcholine causing $50 \%$ inhibition were estimated from the fit of the data to a four parameter logistic fit in Origin ${ }^{\mathrm{TM}}$. From the $\mathrm{EC}_{50}$ values the dose-ratios were computed to construct Schild plots [8]. Concentrations of acetylcholine $>10^{-6} \mathrm{M}$ were not used in control experiments since higher concentrations caused the heart to stop beating. Concentrations of acetylcholine $>10^{-6} \mathrm{M}$ were used in the presence of desloratadine and diphenhydramine since these were found to be antagonists at the muscarinic receptor.

Data are presented as mean \pm SEM. The $n$ values given are for the number of animals used. Data were compared statistically using one-way ANOVA with repeated measures with $p<0.05$ considered significant.

\section{Results}

Left ventricular wall tension was measured using a saline-filled balloon inserted into the left ventricle. The balloon was expanded stepwise to stretch the ventricular wall during the $30 \mathrm{~min}$ equilibration period, until systolic contractions were maximal. As illustrated in Figure 1 the hearts beat rhythmically under sinus control. Typically a heart mounted in the Langendorff apparatus would beat stably for 4 or $6 \mathrm{~h}$, sufficient time to permit multiple acetylcholine concentration response curves to be constructed both in the presence and absence of antihistamine. Heart rate was $>200 \mathrm{bpm}$ in control hearts as indicated in Table 1. Acetylcholine at $10^{-6} \mathrm{M}$ caused an approximate $7 \%$ decrease in 


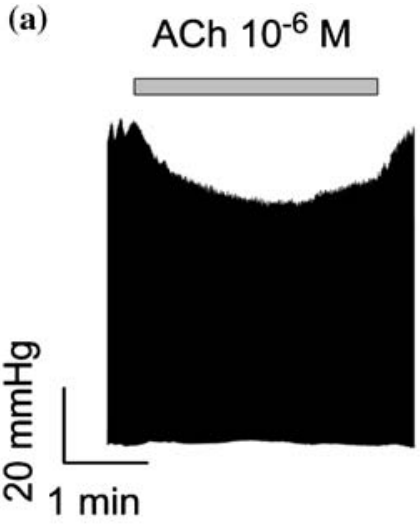

(c) Desloratadine $10^{-6} \mathrm{M}$

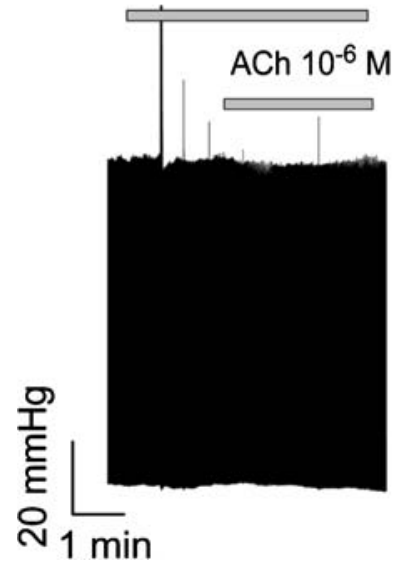

(b) Diphenhydramine $10^{-6} \mathrm{M}$

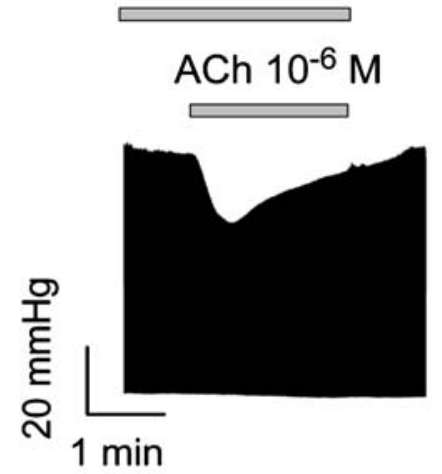

(d) Fexofenadine $10^{-5} \mathrm{M}$

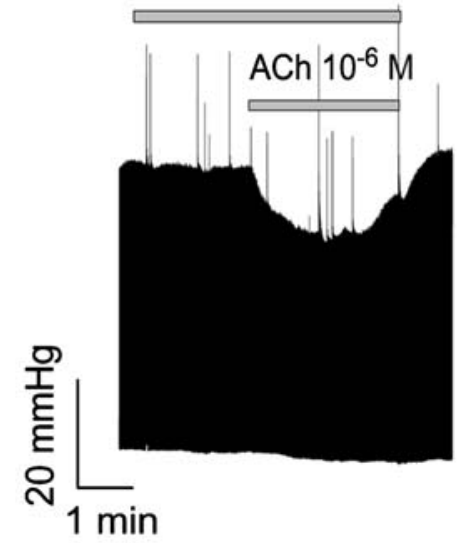

Figure 1. Effects of acetylcholine and antihistamines on cardiac contraction. (a) Acetylcholine $(1 \mu \mathrm{M})$ was perfused into the aorta as described in the Methods section and left ventricular contractions were measured continuously. After switching to perfusion solution containing acetylcholine (indicated by horizontal bar) the peak contraction of the ventricle was reduced reversibly. The effects of three antihistamines on the acetylcholine-induced inhibition are shown in panels b-d. (b) The effect of diphenhydramine ( $1 \mu \mathrm{M}$, upper horizontal bar) on the inhibitory action of $1 \mu \mathrm{M}$ acetylcholine (lower horizontal bar). The effect of acetylcholine is significantly reduced. (c) Desloratadine $(1 \mu \mathrm{M})$ has a similar action on acetylcholine-indued inhibition of contraction. (d) Fexofenadine $(10 \mu \mathrm{M})$ has no effect on contraction. Note the lack of direct of the antihistamines on contraction when applied alone (washin period prior to acetylcholine perfusion). The records shown are from different animals.

Table 1. Effects of antihistamines on heart rate (beats per minute).

\begin{tabular}{lllll}
\hline & $\begin{array}{l}10 \mu \mathrm{M} \\
\text { diphenhydramine } \\
(n=4)\end{array}$ & $\begin{array}{l}3 \mu \mathrm{M} \\
\text { desloratadine } \\
(n=3)\end{array}$ & $\begin{array}{l}10 \mu \mathrm{M} \\
\text { fexofenadine } \\
(n=5)\end{array}$ & $\begin{array}{l}10 \mu \mathrm{M} \\
\text { cetirizine } \\
(n=3)\end{array}$ \\
\hline $\begin{array}{l}\text { Control } \\
\text { After } \\
\text { antihistamine }\end{array}$ & $203 \pm 3$ & $231 \pm 18$ & $234 \pm 10$ & $215 \pm 21$ \\
\hline
\end{tabular}

heart rate from $244 \pm 6$ to $226 \pm 7$ beats per minute $(n=9)$ that was significant $(p<0.05)$. Lower concentrations of acetylcholine did not significantly reduce heart rate. Also, as indicated in Table 1, none of the antihistamines tested had an effect on resting heart rate. As shown in Figure 1a acetylcholine reversibly decreased the systolic contraction by about $40-50 \%$ consistent with the negative 

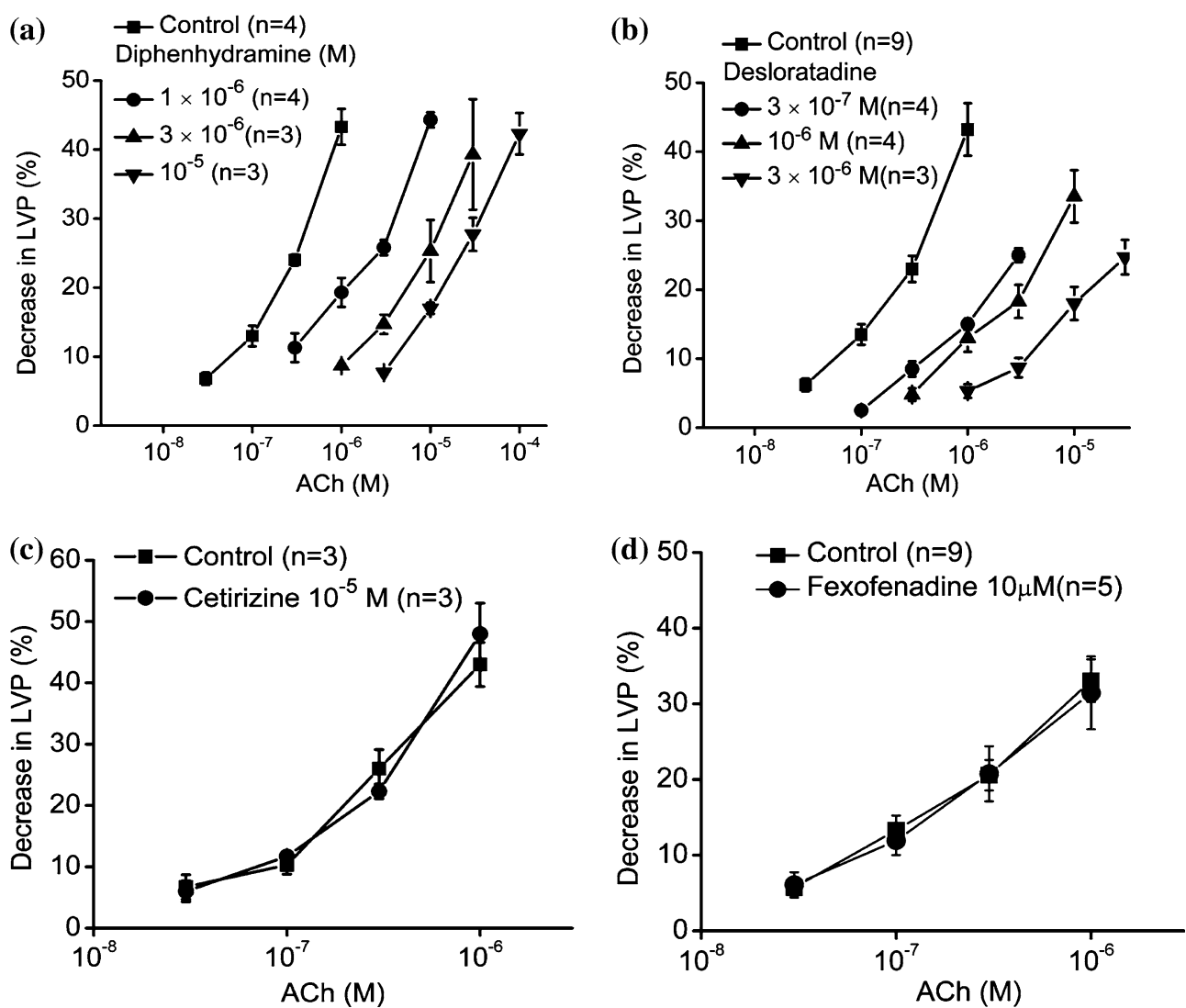

Figure 2. Concentration response relationships for acetylcholine-induced reduction of cardiac contraction: the effects of antihistamines. Concentration response relationships were computed from data similar to that in Figure 1. The percent reduction in peak contractile force was calculated using the peak detect algorithm of Origin ${ }^{\mathrm{TM}}$ and then averaging approximately 300 beats when maximal changes are reached. It was possible to decrease contraction by about $50 \%$ with $1 \mu \mathrm{M}$ acetylcholine in control solution with minimal effects on sino-atrial rate. Higher concentrations of acetylcholine stopped the heart from beating. (a) Diphenhydramine causes parallel shifts in the concentration response relationships to the right with increasing concentrations of diphenhydramine. The lines shown were fit with a four parameter logistic fit in Origin ${ }^{\mathrm{TM}}$. (b) Desloratadine has a similar action to that of diphenhydramine. Panels $\mathrm{c}$ and $\mathrm{d}$ show the lack of effect of fexofenadine and cetirizine on the concentration response relationships. Note that data for only the highest concentration of fexofenadine and cetirizine used $(10 \mu \mathrm{M})$. Data are presented as mean $\pm \mathrm{SEM}$ from three to nine animals (as indicated in the figure).

dromotropic effect of M2 muscarinic receptor activation although as noted earlier higher concentrations were not used in control since they stopped the heart. However, concentrations of acetylcholine greater than $1 \mu \mathrm{M}$ were used in the presence of some antihistamines (diphenhydramine and desloratadine) due to the inhibitory effect of the antihistamine on the actions of acetylcholine. The muscarinic effects of acetylcholine and the antihistamines reversed upon washout of the drugs. The inhibition of the acetylcholine-induced reduction of contraction is illustrated in Figure 1 for diphenhydramine (Figure 1b) and desloratadine (Figure 1c) by the decreased effectiveness of ace- tylcholine $\left(10^{-6} \mathrm{M}\right)$. By contrast, fexofenadine (Figure 1d) and cetirizine (not shown) had no effect on the decrease in contraction induced by acetylcholine. Diphenhydramine, desloratadine, fexofenadine nor cetirizine applied alone had significant actions on contraction as illustrated in Figure 1a-d during the time prior to the perfusion of the heart with acetylcholine. These data were then analyzed for the effects of the antihistamine on the concentration response relationships for acetylcholine. The concentration response data are shown in Figure 2a-d.

Both diphenhydramine $(1,3$ and $10 \mu \mathrm{M}$, Figure $2 \mathrm{a})$ and desloratadine $(0.3,1$ and $3 \mu \mathrm{M}$, 


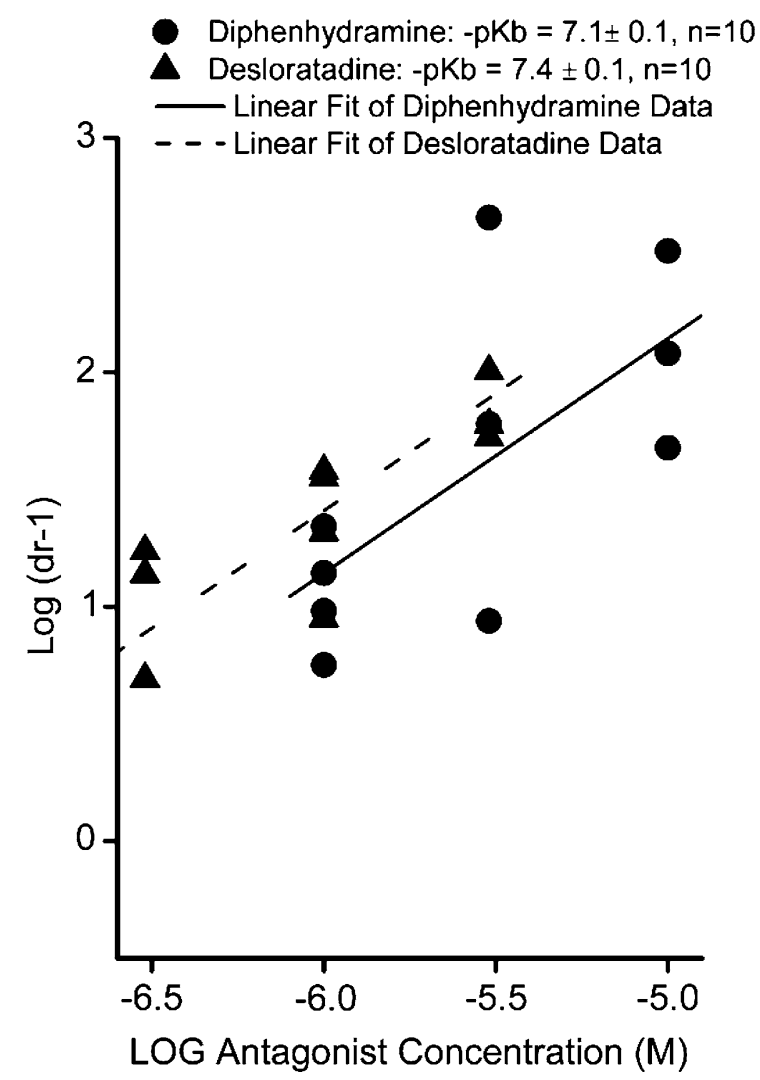

Figure 3. Schild plots are shown in this graph for desloratadine and diphenhydramine. The $Y$ axis is the dose-ratio minus one of the concentrations of acetylcholine giving equal responses in the presence and absence of inhibitor plotted against the logarithm of the inhibitor concentration. The $x$ intercept is the $-\mathrm{pA}_{2}\left(\mathrm{pK}_{b}\right)$. The estimated $\mathrm{pK}_{b}$ are given in the figure. The lines drawn have a slope of 1 . Data are presented as mean $\pm \mathrm{SEM}$.

Figure 2b) caused parallel shifts in the concentration-response relationships to the right. Neither fexofenadine nor cetirizine at $10 \mu \mathrm{M}$ had any effect on the concentration-response relationships for acetylcholine, as shown in Figure $2 \mathrm{c}$ and $\mathrm{d}$, respectively. In two experiments $100 \mu \mathrm{M}$ fexofenadine was shown to have no effect on the acetylcholine induced decreases in contraction (data not shown). The concentration response data for desloratadine and diphenhydramine were then used to construct Schild plots as shown in Figure 3.

Schild analysis [8] for both diphenhydramine (circle, solid line) and desloratadine (triangle, dashed line) yielded linear plots with slopes not significantly different from one. The $\mathrm{pA}_{2}$ calculated from these data were $7.1 \pm 0.1$ and $7.4 \pm 0.1$ for diphenhydramine and desloratadine, respectively.

\section{Discussion}

The cardiovascular effects of antihistamines have been studied more intensely in recent years due to the untoward effect of terfenadine and astemizole to cause cardiac arrhythmias [4]. The arrhythmias, in the case of terfenadine, were caused by the altered pharmacokinetics of the antihistamine in patients when concurrent administration of an antibiotic or antifungal (e.g., erythromycin, ketoconazole) caused elevated plasma levels of the antihistamine [9]. The high levels of antihistamine inhibited HERG potassium channels in the heart and resulted in Torsades de pointe, a serious arrhythmia [4]. All of the second generation compounds are now tested for effects on the HERG channel and none interact significantly. However, another potential cardiac interaction is with muscarinic receptors in the heart. The potential for antihistamines to interact with muscarinic receptors is well known [5]. We found that one of the newest antihistamines tested, desloratadine, competitively inhibited M2 muscarinic receptors in rat heart. Two other newer compounds, fexofenadine and cetirizine did not interact with these receptors.

Fexofenadine and cetirizine had no effect on the concentration response relationships for acetylcholine demonstrating a lack of interaction with M2 receptors. By contrast, both diphenhydramine and desloratadine competitively inhibited acetylcholine-induced inhibition of cardiac contraction. Desloratadine and diphenhydramine inhibited M2 muscarinic receptors with estimated $\mathrm{K}_{i}$ of 0.04 and $0.08 \mu \mathrm{M}$. Kubo et al. [5] measured the $\mathrm{K}_{i}$ for diphenhydramine against QNB binding in the cerebral cortex, a ligand for all muscarinic receptor subtypes, of $0.28 \mu \mathrm{M}$. Cardelus et al. [6] estimated the potency of desloratadine of approximately $0.2 \mu \mathrm{M} \quad\left(\mathrm{pA}_{2}=6.7 \pm 0.1\right)$ against muscarinic-induced contraction of rabbit iris, presumably a M3 mediated response. Kubo et al. [5] found that hydroxyzine had a $\mathrm{K}_{i}$ of $3.8 \mu \mathrm{M}$ against muscarinic receptors in the cerebral cortex using radioligand binding assays. It is interesting that the parent antihistaminic compound of desloratadine, loratadine, does not inhibit muscarinic receptors [10]. The question then arises; why do some 
<smiles>NC12CCCCCC1CC(Cc1cccc3cc(Cl)ccc13)C2</smiles>

desloratadine<smiles>CCOC(=O)N1CCN(C(=C2CCc3cc(Cl)ccc32)c2ncccc2C)CC1</smiles><smiles>CCC(CO)c1ccc(C(C)(C)C(=O)O)cc1</smiles><smiles>OCCOCCN1CCCCC1(c1ccccc1)N1CCN(CCO)CC1</smiles>

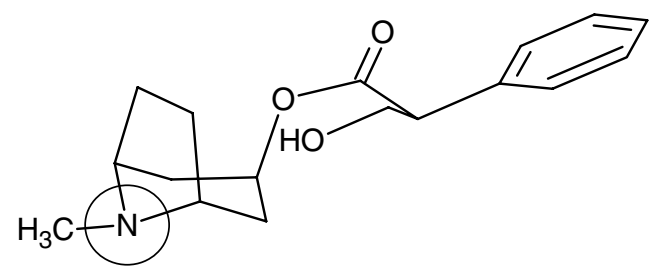

atropine<smiles>CN1CCN(CCOC(c2ccccc2)c2ccccc2)CC1</smiles>

diphenhydramine

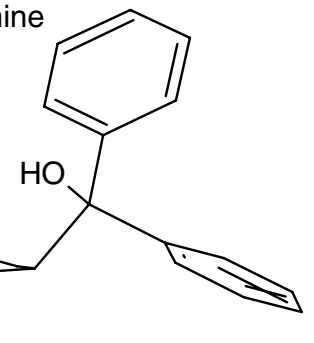

Figure 4. The structures of each of the antihistamines used in this study including those for hydroxyzine and loratadine and the prototypical muscarinic antagonist atropine are shown. Circles are drawn around the nitrogen in each antihistamine that is in a configuration similar to the tropine nitrogen of atropine (also circled). Substitutions on the nitrogen circled in the antihistamines may be important in determining the antihistamine's antimuscarinic activity as discussed in the text. 
antihistamines inhibit muscarinic receptors while other closely related compounds do not?

The structures of several antihistamines are shown in Figure 4 along with the structure for the prototypical muscarinic antagonist, atropine. There are two common features of all the antihistamines, (1) an unsaturated ring structure consisting of several rings in various conformations and (2) one or two nitrogens in an ethanolamine (diphenhydramine), a piperidine (fexofenadine, loratadine, desloratadine) or a piperazine (cetirizine, hydroxyzine) side group. One nitrogen (circled in the antihistamines in Figure 4) is in a similar configuration as the tropine nitrogen in atropine. The compounds with the highest affinity for muscarinic receptors are those compounds with no or non-polar substitutions on the nitrogen (desloratadine and diphenhydramine). Loratadine is a carboxylate, and fexofenadine and cetirizine have acetic acid moieties in the side chain attached to the nitrogen circled in Figure 4. These drugs have no measurable antimuscarinic properties. Zhang et al. [11] suggested that for ebastine the chirality of the benzhydryl chiral center was important for antihistaminic potency with the $\mathrm{R}$ form most potent. The $\mathrm{R}$ form had the least antimuscarinic action. In addition substitution of a single ketone or alcohol oxygen in the phenyl butanol side chain did not greatly alter muscarinic potency, although it did enhance antihistaminic potency. Therefore we conclude that substitution on the nitrogen in the piperidine or piperazine side chain with polar or electronegative groups decreases potency as an antagonist at muscarinic receptors. Desloratadine and diphenhydramine have the highest affinity for muscarinic receptors in the heart and the least polar side chains of the compounds tested. Fexofenadine and cetirizine do not interact with the muscarinic receptors in the heart. Therefore, of the compounds tested, only diphenhydramine and desloratadine would be expected to have cardiovascular effects due to muscarinic receptor blockade in the heart.

\section{Acknowledgement}

This work was supported by a grant from Aventis.

\section{References}

1. Nicolas J.M., The metabolic profile of second-generation antihistamines. Allergy 55(Suppl 60): 46-52, 2000.

2. Nathan R.A., Pharmacotherapy for allergic rhinitis: a critical review of leukotriene receptor antagonists compared with other treatments. Ann. Allergy Asthma Immunol. 90: 182-190, 2003.

3. Taglialatela M., Pannaccione A., Castaldo P., Giorgio G., Zhou Z., January C.T., Genovese A., Marone G. and Annunziato L., Molecular basis for the lack of HERG K ${ }^{+}$ channel block-related cardiotoxicity by the $\mathrm{H} 1$ receptor blocker cetirizine compared with other second-generation antihistamines. Mol. Pharmacol. 54: 113-121, 1998.

4. Taglialatela M., Castald P., Pannaccion A., Giorgi G., Genoves A., Maron G. and Annunziat L., Cardiac ion channels and antihistamines: possible mechanisms of cardiotoxicity. Clin. Exp. Allergy 29(Suppl 3): 182-189, 1999.

5. Kubo N., Shirakawa O., Kuno T. and Tanaka C., Antimuscarinic effects of antihistamines: quantitative evaluation by receptor-binding assay. Jpn. J. Pharmacol. 43: 277-282, 1987.

6. Cardelus I., Anton F., Beleta J. and Palacios J.M., Anticholinergic effects of desloratadine, the major metabolite of loratadine, in rabbit and guinea-pig iris smooth muscle. Eur. J. Pharmacol. 374: 249-254, 1999.

7. Vergely C., Perrin-Sarrado C., Clermont G. and Rochette L., Postischemic recovery and oxidative stress are independent of nitric-oxide synthases modulation in isolated rat heart. J. Pharmacol. Exp. Ther. 303: 149-157, 2002.

8. Arunlakshana O. and Schild H.O., Some quantitative uses of drug antagonists. Br. J. Pharmacol. 14: 48-58, 1959.

9. Presa J., H1 Antihistamines: a review. Alergol. Immunol. Clin. 14: 300-312, 1999.

10. Handley D.A., McCullough J.R., Fand Y., Wright S.E. and Smith E.R., Descarboethoxyloratadine, a metabolite of loratadine, is a superior antihistamine. Ann. Allergy Asthma Immunol. 78: P164, 1997.

11. Zhang M.Q., Walczynski K. and Timmerman H., A steric approach for the design of antihistamines with low muscarinic receptor antagonism. Inflamm. Res. 44(Suppl 1): S90-S91, 1995. 